\title{
Selective generation and amplification of RKKY interactions by P-N interface
}

\author{
Shu-Hui Zhang ${ }^{1,2}$, Jia-Ji Zhu ${ }^{3}$, Wen Yang ${ }^{1}$ * and Kai Chang ${ }^{4,5}$, \\ ${ }^{1}$ Beijing Computational Science Research Center, Beijing 100193, China \\ ${ }^{2}$ College of Science, Beijing University of Chemical Technology, Beijing 100029, China \\ ${ }^{3}$ Institute for quantum information and spintronics, School of Science, \\ Chongqing University of Posts and Telecommunications, Chongqing 400065, China \\ ${ }^{4}$ SKLSM, Institute of Semiconductors, Chinese Academy of Sciences, P.O. Box 912, Beijing 100083, China and \\ ${ }^{5}$ Synergetic Innovation Center of Quantum Information and Quantum Physics, \\ University of Science and Technology of China, Hefei, Anhui 230026, China
}

\begin{abstract}
We propose a physical mechanism to generate and selectively amplify anisotropic Rudermann-Kittel-KasuyaYosida (RKKY) interactions between two local spins. The idea is to combine the deflection of the carrier velocity by a P-N interface and the locking of this velocity to the carrier spin orientation via spin-orbit coupling. We provide analytical and numerical results to demonstrate this mechanism on the surface of a topological insulator $\mathrm{P}-\mathrm{N}$ junction. This work identifies the P-N interface as a second knob which, together with the carrier density, enables independent control of the strength and anisotropy of the RKKY interaction over a wide range. These findings may be relevant to scalable quantum computation and two-impurity quantum criticality.
\end{abstract}

PACS numbers: 73.40.Lq, 75.30.Hx, 72.80.Vp, 73.23.Ad

\section{INTRODUCTION}

The carrier-mediated Rudermann-Kittel-Kasuya-Yosida (RKKY) interaction ${ }^{1-4}$ between local spins has been known for more than sixty years as an important mechanism for the broadening of nuclear spin resonance lines, magnetic ordering, and spin glass behaviors in magnetic metals, alloys, and semiconductors. It characterizes the magnetic response of the itinerant carriers and finds interesting applications, e.g., it enables entanglement between spatially separated spins in quantum $\operatorname{dot}^{5-9}$ for scalable quantum computation 1011 and allows magnetic ordering of magnetically doped materials for spintronics ${ }^{12}\left[14\right.$ and new topological phases $\left.{ }^{15} \cdot 17\right]$. For a pair of magnetic atoms, it competes with the Kondo effect and gives rise to rich physics $\$ \frac{180}{20}$ that have been of considerable theoretical and experimental interest for decades $21+24$. For these applications, selective amplification of different interaction terms (beyond the isotropic Heisenberg-like term) is highly desirable.

Recently, the RKKY interaction was studied in many materials, e.g., dilute ferromagnetic semiconductors (see Ref. 4 for a review), graphene $e^{25 \sqrt[30]{3}}$, Dirac and Weyl semimetal ${ }^{\sqrt{3132}}$, and the surface of topological insulators (TIs) ${ }^{33}+39$. The successful measurement $\left.{ }^{40}-43\right]$ of the RKKY interaction with atomic-scale spatial resolution ${ }^{42 / 43}$ opens a new playground for engineering this interaction. Interesting theoretical and experimental works for amplifying the isotropic Heisenberg-like term have been reported ${ }^{22 / 44}[55]$, but selective generation and amplification of anisotropic terms remains elusive. The general consensus is that these terms originate from the spin-orbit coupling of the carriers, so tuning these terms requires tuning the symmetry of the spin-orbit coupling (e.g., combining the Rashba and Dresselhaus terms ${ }^{56}$ or the warping effect of TI surface state ${ }^{35}$ ). The latter is determined by the crystal symmetry and sample orientation, so it has no (or very limited) tunability.

Here we propose a physical mechanism to generate and selectively amplify anisotropic interaction terms between two local spins $\hat{\mathbf{S}}_{1}$ and $\hat{\mathbf{S}}_{2}$. The idea is that these terms arise from the spin orientation of carriers traveling between $\hat{\mathbf{S}}_{1}$ and $\hat{\mathbf{S}}_{2}$, so the P-N interface can deflect the velocity and hence the spin (via spin-orbit coupling) of those carriers to achieve controlled generation of anisotropic terms. The negative refraction of carriers across the P-N interface ${ }^{57,60}$ further allows selective amplification of these terms. This physical mechanism is applicable to the $\mathrm{P}-\mathrm{N}$ junction of any material with spin-orbit coupling. For specificity, here we demonstrate this mechanism by both analytical and numerical results on the surface of a TI P-N junction, which forms the basis of several interesting proposal $\frac{58161,63}{63}$ and was recently fabricated $\frac{64 \sqrt[67]{67}}{\text { with }}$ atomically abrupt interfaces ${ }^{67}$. Ever since the discovery of the RKKY interaction, extensive efforts have been devoted to this interaction, the vast majority of which focus on its manipulation in uniform materials via the carrier density. Here, our work identifies the P-N interface as a second knob which, together with the carrier density, enables independent control of the strength and anisotropy of the RKKY interaction. These findings mark a step towards engineering RKKY interactions in spin-orbit coupled systems for the nonlocal control of spin and entanglement.

This paper is organized as follows. In Sec. II, we give an intuitive physical picture for the origin of the anisotropic terms. In Sec. III, we provide analytical and numerical results for engineering the RKKY interactions on the surface of TI $\mathrm{P}-\mathrm{N}$ junctions. Finally we summarize our findings in Sec. IV.

\section{PHYSICAL PICTURE}

We consider two local spins $\hat{\mathbf{S}}_{1}$ (located at $\mathbf{R}_{1}$ ) and $\hat{\mathbf{S}}_{2}$ (located at $\mathbf{R}_{2}$ ) coupled to itinerant carriers via the contact exchange interaction $-\lambda \sum_{i} \hat{\mathbf{S}}_{i} \cdot \hat{\boldsymbol{\sigma}} \delta\left(\hat{\mathbf{r}}-\mathbf{R}_{i}\right)$, where $\hat{\boldsymbol{\sigma}}$ are Pauli matrices for the carrier spin. At low temperature, the carriers mediate an effective interaction ${ }^{1 / 368}$ between $\hat{\mathbf{S}}_{1}$ and $\hat{\mathbf{S}}_{2}$ :

$$
\hat{H}_{\mathrm{RKKY}}=-\frac{\lambda^{2}}{\pi} \operatorname{Im} \int_{-\infty}^{E_{F}} \hat{K}(E) d E,
$$


where $E_{F}$ is the Fermi energy, $\operatorname{Im} \hat{O} \equiv\left(\hat{O}-\hat{O}^{\dagger}\right) /(2 i)(\forall \hat{O})$, and

$$
\hat{K}(E)=\operatorname{Tr}\left[\hat{\boldsymbol{\sigma}} \cdot \hat{\mathbf{S}}_{1} \hat{G}\left(\mathbf{R}_{1}, \mathbf{R}_{2} ; E\right) \hat{\boldsymbol{\sigma}} \cdot \hat{\mathbf{S}}_{2} \hat{G}\left(\mathbf{R}_{2}, \mathbf{R}_{1} ; E\right)\right] .
$$

Here the carrier propagator (retarded Green's function) $\hat{G}\left(\mathbf{r}_{2}, \mathbf{r}_{1} ; E\right)$ in real space is still an operator acting on the carrier spin and the trace is taken over the carrier spin.

Equation (2) shows that the RKKY interaction arises from two steps: (i) The carrier travels from $\hat{\mathbf{S}}_{1}$ to $\hat{\mathbf{S}}_{2}$, as described by $\hat{G}\left(\mathbf{R}_{2}, \mathbf{R}_{1} ; E\right)$, and undergoes the interaction $\hat{\boldsymbol{\sigma}} \cdot \hat{\mathbf{S}}_{2} ;$ (ii) The carrier travels from $\hat{\mathbf{S}}_{2}$ back to $\hat{\mathbf{S}}_{1}$, as described by $\hat{G}\left(\mathbf{R}_{1}, \mathbf{R}_{2} ; E\right)$, and undergoes the interaction $\hat{\boldsymbol{\sigma}} \cdot \hat{\mathbf{S}}_{1}$, which restores the initial spin state of the carrier. This process mediates an effective interaction $\sim \hat{K}(E)$ between $\hat{\mathbf{S}}_{1}$ and $\hat{\mathbf{S}}_{2}$. The sum of the contributions from all the occupied carrier states gives the total RKKY interaction in Eq. (1). When the distance $R \equiv\left|\mathbf{R}_{2}-\mathbf{R}_{1}\right|$ exceeds the Fermi wavelength of the carriers, stationary phase approximation ${ }^{6970}$ shows that the energy integral in Eq. (1) is dominated by the contribution near the Fermi leve ${ }^{48 / \pi}$ : $\hat{H}_{\mathrm{RKKY}} \propto \hat{K}\left(E_{F}\right) / R$, so the strength (anisotropy) of the RKKY interaction is determined by the magnitude (spin polarization) of the carrier propagator on the Fermi level.

In a $d$-dimensional uniform system, the propagators $\hat{G}\left(\mathbf{R}_{2}, \mathbf{R}_{1} ; E_{F}\right)$ and $\hat{G}\left(\mathbf{R}_{1}, \mathbf{R}_{2} ; E_{F}\right)$ mimic an outgoing spherical wave and decays as $1 / R^{(d-1) / 2}$ to conserve the total probability current, so the RKKY interaction exhibits "universal" $1 / R^{d}$ decay $\sqrt{55}$, as found in many previous studies. When the carrier's density of states on the Fermi level vanishes, the RKKY interaction may decay even faster, e.g., when the Fermi level locates at the Dirac/Weyl point, it follows $1 / R^{3}$ decay in graphene $e^{252728}$ and on the surface of TIs ${ }^{33}+36$, and follows $1 / R^{5}$ decay in Dirac/Weyl semimetals $s^{31 / 32}$.

Next we turn to the anisotropy of the RKKY interaction. Without spin-orbit coupling, the carrier propagator is spinindependent, so $\hat{K}(E)$ only contains the isotropic Heisenberg term $\hat{\mathbf{S}}_{1} \cdot \hat{\mathbf{S}}_{2}$. With spin-orbit coupling, the anisotropy originates intuitively as follows. In a uniform system, carriers going from $\hat{\mathbf{S}}_{1}$ to $\hat{\mathbf{S}}_{2}$ have a group velocity along $\mathbf{R}_{2}-\mathbf{R}_{1}$, while those going from $\hat{\mathbf{S}}_{2}$ back to $\hat{\mathbf{S}}_{1}$ have an opposite group velocity [blue arrows in Fig. [1(a)]. Usually, opposite group velocities $\pm \mathbf{v}$ amounts to opposite momenta $\pm \mathbf{k}$, which in turn are locked to opposite spin orientations $\pm \mathbf{b}(\mathbf{k})$ via a generic spin-orbit coupling $\hat{\boldsymbol{\sigma}} \cdot \mathbf{b}( \pm \mathbf{k})= \pm \hat{\boldsymbol{\sigma}} \cdot \mathbf{b}(\mathbf{k})$ that preserves timereversal symmetry. For clarity, we use a unit vector $\mathbf{e}(-\mathbf{e})$ [red arrows in Fig. 11(a)] for the spin orientation of those carriers traveling from $\hat{\mathbf{S}}_{1}$ to $\hat{\mathbf{S}}_{2}$ (from $\hat{\mathbf{S}}_{2}$ back to $\hat{\mathbf{S}}_{1}$ ), and $|\mathbf{n}\rangle$ for the spin-up state along the vector $\mathbf{n}$, then $\hat{G}\left(\mathbf{R}_{2}, \mathbf{R}_{1} ; E_{F}\right) \propto|\mathbf{e}\rangle\langle\mathbf{e}|$ and $\hat{G}\left(\mathbf{R}_{1}, \mathbf{R}_{2} ; E_{F}\right) \propto|-\mathbf{e}\rangle\langle-\mathbf{e}|$, so Eq. 2) gives

$$
\hat{K}\left(E_{F}\right) \propto\left(\langle\mathbf{e}|\hat{\boldsymbol{\sigma}}|-\mathbf{e}\rangle \cdot \hat{\mathbf{S}}_{1}\right) \times\left(\langle-\mathbf{e}|\hat{\boldsymbol{\sigma}}| \mathbf{e}\rangle \cdot \hat{\mathbf{S}}_{2}\right)=\hat{S}_{1, \mathbf{e}}^{+} \hat{S}_{2, \mathbf{e}}^{-},
$$

where $\hat{S}_{\mathbf{e}}^{+}\left(\hat{S}_{\mathbf{e}}^{-}\right)$is the spin raising (lowering) operator that increases (decreases) $\hat{\mathbf{S}} \cdot \mathbf{e}$ by one. Physically, this interaction comes from the following two-step process [cf. Fig. 11a)]:

(i) A carrier with group velocity $\mathbf{v}$ and spin $|\mathbf{e}\rangle$ travels from $\hat{\mathbf{S}}_{1}$ to $\hat{\mathbf{S}}_{2}$ and interacts with $\hat{\mathbf{S}}_{2}$. This interaction reverses the carrier velocity to $\mathbf{- v}$ and the carrier spin to $|-\mathbf{e}\rangle$. It also increases $\hat{\mathbf{S}}_{2} \cdot \mathbf{e}$ by one to conserve the total spin along $\mathbf{e}$.
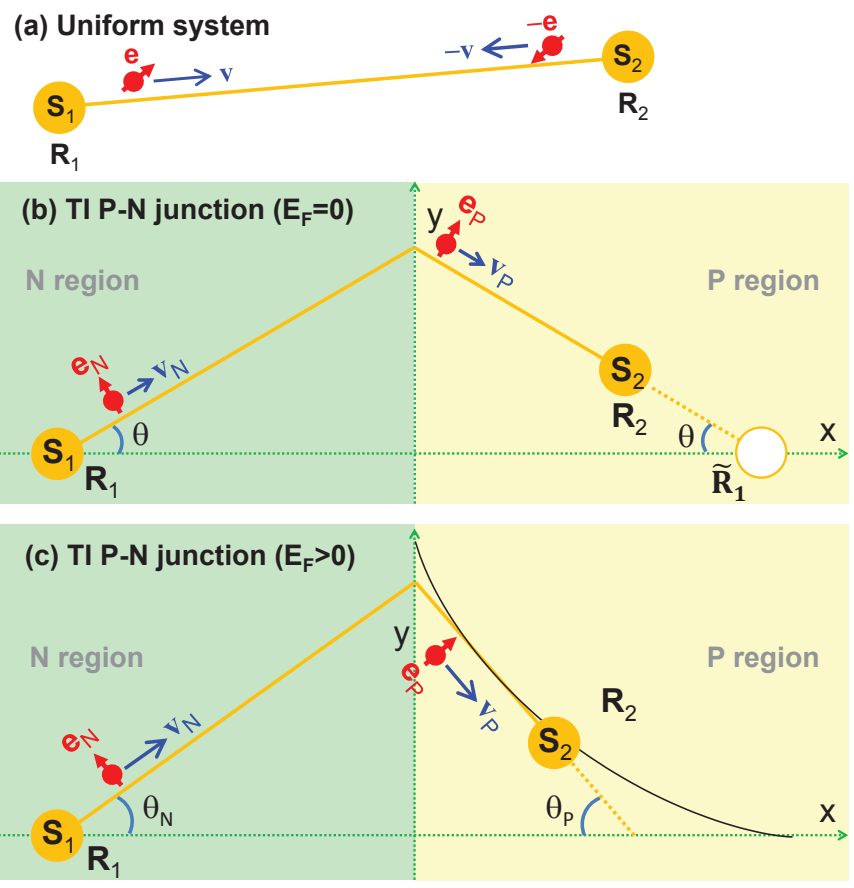

FIG. 1. Group velocity (blue arrows) and spin orientation (red arrows) of itinerant carriers traveling along a classical trajectory (orange line) that connects the two local spins (a) in uniform system, or on the surface of (b) symmetric or (c) asymmetric TI P-N junction. The black curve in (c) indicates the caustics above the $x$ axis.

(ii) This carrier travels from $\hat{\mathbf{S}}_{2}$ back to $\hat{\mathbf{S}}_{1}$ and interacts with $\hat{\mathbf{S}}_{1}$. This interaction restores the carrier group velocity back to $\mathbf{v}$ and the carrier spin back to $|\mathbf{e}\rangle$. It also decreases $\hat{\mathbf{S}}_{1} \cdot \mathbf{e}$ by one to conserve the total spin along $\mathbf{e}$.

The entire process leaves the carrier spin intact, but increases $\hat{\mathbf{S}}_{2} \cdot \mathbf{e}$ by one and decreases $\hat{\mathbf{S}}_{1} \cdot \mathbf{e}$ by one. Explicitly,

$$
\hat{S}_{1, \mathbf{e}}^{+} \hat{S}_{2, \mathbf{e}}^{-}=\hat{\mathbf{S}}_{1} \cdot \hat{\mathbf{S}}_{2}-\left(\mathbf{e} \cdot \hat{\mathbf{S}}_{1}\right)\left(\mathbf{e} \cdot \hat{\mathbf{S}}_{2}\right)+i \mathbf{e} \cdot\left(\hat{\mathbf{S}}_{1} \times \hat{\mathbf{S}}_{2}\right)
$$

contains the isotropic Heisenberg term and the anisotropic Ising and Dzyaloshinskii-Moriya terms, as found previously in many materials, such as Dirac and Weyl semimetal $\$ 31 / 32$ and the surface of TIs $s^{33}$.39.

Therefore, the outgoing propagation (spin polarization) of the carriers traveling between $\hat{\mathbf{S}}_{1}$ and $\hat{\mathbf{S}}_{2}$ generates the $1 / R^{d}$ decay (anisotropic terms) of the RKKY interaction. Usually, the spin polarization axis $\mathbf{e}$ is determined by $\mathbf{R}_{2}-\mathbf{R}_{1}$ and the spin-orbit coupling [e.g., $\mathbf{e} \propto \mathbf{e}_{z} \times\left(\mathbf{R}_{2}-\mathbf{R}_{1}\right)$ on a TI surface], making selective control of different interactions difficult. Interestingly, the P-N interface can change both behaviors, so it can generate and selectively amplify anisotropic RKKY interactions. This physical mechanism is applicable to any material with spin-orbit coupling. Here for clarity, we illustrate this mechanism for the surface of a TI P-N junction. 


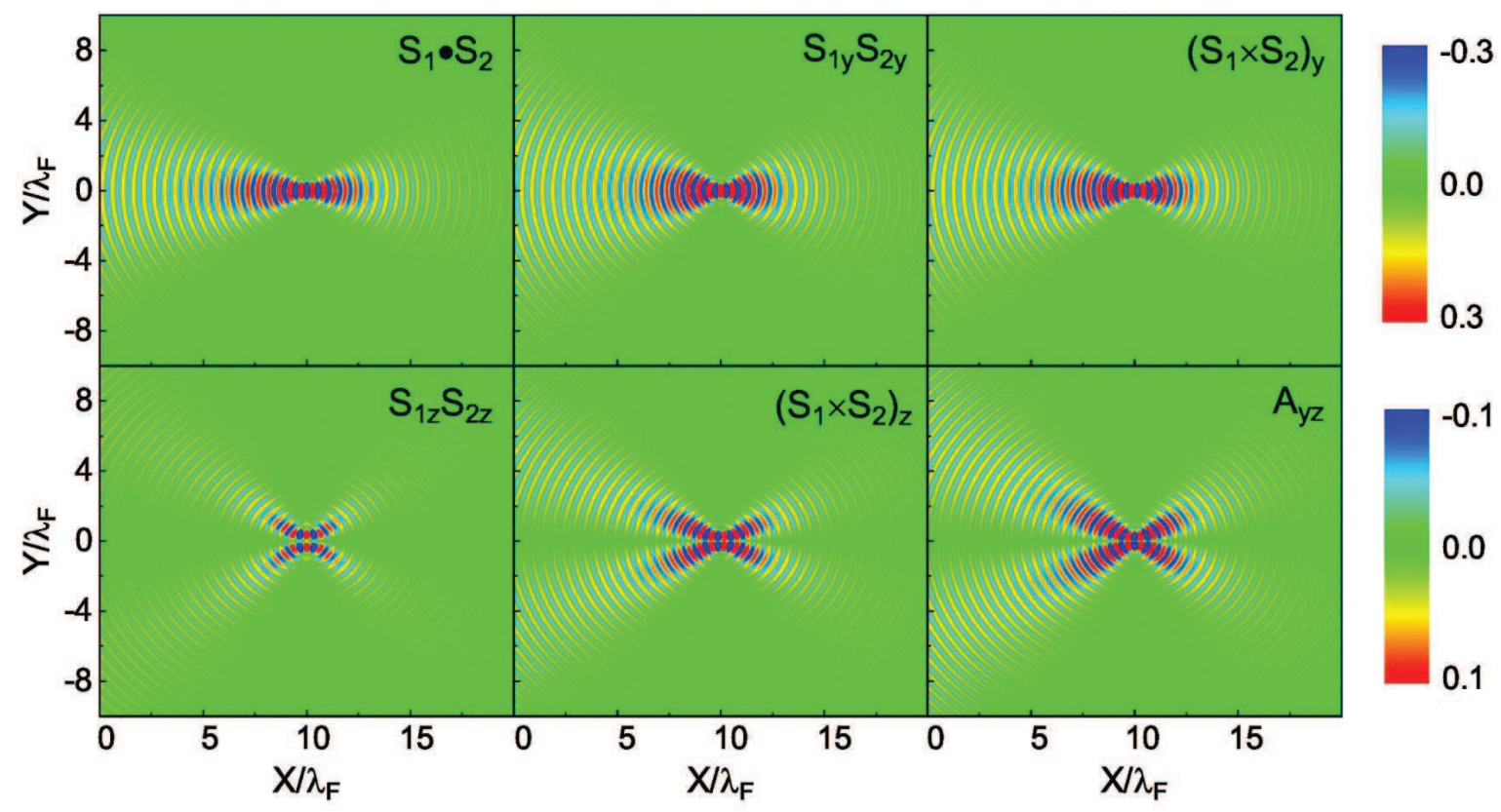

FIG. 2. Spatial map of different RKKY interactions [unit: $J_{0} /(2 a)$ ] vs. $\mathbf{R}_{2}=(X, Y)$ in a symmetric P-N junction, where $A_{\alpha \beta} \equiv \hat{S}_{1}^{\alpha} \hat{S}_{2}^{\beta}+\hat{S}_{1}^{\beta} \hat{S}_{2}^{\alpha}$, the first spin is fixed at $\mathbf{R}_{1}=(-a, 0)=\left(-10 \lambda_{F}, 0\right)$, and $J_{0} \equiv \lambda^{2} q_{F}^{2} /\left(8 \pi^{3} v_{F}\right)$.

\section{RKKY INTERACTION IN TI P-N JUNCTIONS}

The TI is a new class of quantum matter with extraordinary surface carriers (in the $x y$ plane) as described by 72173

$$
\hat{H}_{0}=v_{F} \hat{\boldsymbol{\sigma}} \cdot\left(\mathbf{e}_{z} \times \hat{\mathbf{p}}\right),
$$

which gives gapless linear dispersion $E_{ \pm}(\mathbf{q})= \pm v_{F}|\mathbf{q}|$ with group velocity $\mathbf{v}_{ \pm}= \pm v_{F} \mathbf{q} /|\mathbf{q}|$. The corresponding eigenstate is $e^{i \mathbf{q} \cdot \mathbf{r}}\left|\mathbf{e}_{z} \times \mathbf{v}_{ \pm}\right\rangle$, so the spin orientation of a carrier moving with group velocity $\mathbf{v}$ is locked to $\mathbf{e}_{z} \times \mathbf{v}$. Very recently, there were remarkable progresses in fabricating TIbased nanostructures ${ }^{64}$ 67/74. In particular, the TI P-N junctions were recently fabricated ${ }^{64}$ with atomically abrupt interfaces ${ }^{67}$. Previous theoretical works focus on their interesting electronic properties, such as gapless junction states ${ }^{61}$, birefringent spin lens ${ }^{58}$, spin filtering ${ }^{62}$, and spin-based MachZehnder interferometry ${ }^{63}$. Here we explore a very different application: the selective amplification of different RKKY interactions. This may be relevant to the application of TIs to spintronics and quantum computing.

The surface carriers of a TI P-N junction is described by

$$
\hat{H}=\hat{H}_{0}+\operatorname{sgn}(x) V_{0}
$$

with Fermi energy $E_{F} \in\left[-V_{0}, V_{0}\right]$. The left region $(x<0)$ is N-type with electron Fermi momentum $q_{N} \equiv\left(V_{0}+E_{F}\right) / v_{F}$, while the right region $(x>0)$ is P-type with hole Fermi momentum $q_{P} \equiv\left(V_{0}-E_{F}\right) / v_{F}$. Without losing generality, we assume $\hat{\mathbf{S}}_{1}$ locates at $\mathbf{R}_{1}=(-a, 0)$ in the $\mathrm{N}$ region and $\hat{\mathbf{S}}_{2}$ locates at $\mathbf{R}_{2}=(X, Y)$ in the $\mathrm{P}$ region.
A. Symmetric P-N junction $\left(E_{F}=0\right)$

Here the $\mathrm{N}$ region and the $\mathrm{P}$ region have the same Fermi momenta $q_{N}=q_{P}=q_{F} \equiv V_{0} / v_{F}$ and Fermi wavelength $\lambda_{F} \equiv 2 \pi / q_{F}$. As shown in Fig. 11(b), an electron incident from the $\mathrm{N}$ region is refracted by the $\mathrm{P}-\mathrm{N}$ interface. The incident and refractive trajectories are mirror symmetric about the P-N interface, so electrons emanating from $\hat{\mathbf{S}}_{1}$ will be refocused by the P-N interface onto the focal point $\tilde{\mathbf{R}}_{1} \equiv(a, 0)$ [empty circle in Fig. 11.b)]. This is the well-known negative refraction of electrons across a P-N interface ${ }^{575960}$, the electronic analog to its optical counterpart ${ }^{75}$.

On uniform TI surface, the RKKY interaction has rotational symmetry around the axis $\mathbf{e} \propto \mathbf{e}_{z} \times\left(\mathbf{R}_{2}-\mathbf{R}_{1}\right)$, which only allows three terms [Eq. [3] ] that conserve the total spin along e. On the surface of TI P-N junction, this rotational symmetry is broken, so all interactions are allowed: the Heisenberg term $\hat{\mathbf{S}}_{1} \cdot \hat{\mathbf{S}}_{2}$, the Ising terms $\left(\hat{S}_{1 y} \hat{S}_{2 y}\right.$ and $\left.\hat{S}_{1 z} \hat{S}_{2 z}\right)$, the Dzyaloshinskii-Moriya terms $\hat{D}_{x}, \hat{D}_{y}, \hat{D}_{z}$, and $\hat{A}_{x y}, \hat{A}_{x z}, \hat{A}_{y z}$, where $\hat{\mathbf{D}} \equiv \hat{\mathbf{S}}_{1} \times \hat{\mathbf{S}}_{2}$ and $\hat{A}_{\alpha \beta}$ is the element of the symmetric tensor operator $\mathbb{A} \equiv \hat{\mathbf{S}}_{1} \hat{\mathbf{S}}_{2}+\hat{\mathbf{S}}_{2} \hat{\mathbf{S}}_{1}$. Surprisingly, numerical simulations show that only six terms (Fig. 2) are appreciable, while other terms are smaller by two orders of magnitudes (not shown), suggesting the existence of a hidden symmetry. Figure 2 further shows that all the terms are strongly enhanced when $\mathbf{S}_{2}$ locates near the focal point and different terms exhibit distinct angular dependencies. Next, we present analytical expressions and a physical picture for these features.

For $\hat{\mathbf{S}}_{2}$ far from the focal point $\left(\left|\mathbf{R}_{2}-\tilde{\mathbf{R}}_{1}\right| \gtrsim \lambda_{F}\right)$, the propagator from $\hat{\mathbf{S}}_{1}$ to $\hat{\mathbf{S}}_{2}$ is dominated by the classical trajectory [solid orange line in Fig. 11 b)], as characterized by an incident angle $\theta \equiv \tan ^{-1}[Y /(a-X)]$, transmission amplitude $t=\cos \theta$, 
and trajectory length $R_{N}=a / \cos \theta\left(R_{P}=X / \cos \theta\right)$ in the $\mathrm{N}$ (P) region. In the $N(\mathrm{P})$ region, the carrier group velocity is parallel (anti-parallel) to the momentum, so the propagation phase is $q_{F} R_{N}\left(-q_{F} R_{P}\right)$. The total propagation phase is

$$
\Phi \equiv q_{F}\left(R_{N}-R_{P}\right)=\operatorname{sgn}(a-X) q_{F}\left|\mathbf{R}_{2}-\tilde{\mathbf{R}}_{1}\right| .
$$

Using stationary phase approximation $\frac{69}{7176}$ gives (see Appendix A)

$$
\hat{G}\left(\mathbf{R}_{2}, \mathbf{R}_{1}, E_{F}\right) \approx t \frac{q_{F}}{i v_{F}} \frac{e^{i \Phi}}{\sqrt{2 \pi i \Phi}}\left|\mathbf{e}_{\mathrm{P}}\right\rangle\left\langle\mathbf{e}_{\mathrm{N}}\right|
$$

where the spin orientations $\mathbf{e}_{\mathrm{N}}, \mathbf{e}_{\mathrm{P}}$ are indicated by red arrows in Fig. 1(b). The propagator from $\hat{\mathbf{S}}_{2}$ back to $\hat{\mathbf{S}}_{1}$ is obtained from Eq. (4) by time reversal of the spin (i.e., $\left.\left|\mathbf{e}_{\mathrm{P}}\right\rangle\left\langle\mathbf{e}_{\mathrm{N}}|\rightarrow|-\mathbf{e}_{\mathrm{N}}\right\rangle\left\langle-\mathbf{e}_{\mathrm{P}}\right|\right)$. The RKKY interaction is dominated by the contribution on the Fermi level ${ }^{48 / 71}$ :

$$
\hat{H}_{\mathrm{RKKY}} \approx \frac{J^{2} v_{F}}{2 \pi\left(R_{N}+R_{P}\right)} \operatorname{Re} \hat{K}\left(E_{F}\right) .
$$

Using the explicit expressions for the propagators gives

$$
\hat{H}_{\mathrm{RKKY}} \approx-\frac{J_{0} t^{2}}{R_{N}+R_{P}} \frac{2 \pi}{\Phi} \operatorname{Im}\left(e^{2 i \Phi} \hat{S}_{1, \mathbf{e}_{\mathrm{N}}}^{-} \hat{S}_{2, \mathbf{e}_{\mathrm{P}}}^{+}\right)
$$

where $J_{0} \equiv \lambda^{2} q_{F}^{2} /\left(8 \pi^{3} v_{F}\right)$ and $\hat{S}_{\mathbf{e}}^{+}\left(\hat{S}_{\mathbf{e}}^{-}\right)$increases (decreases) $\hat{\mathbf{S}} \cdot \mathbf{e}$ by one. This interaction arises from the following process [cf. Fig. 11 b)]:

(1) A carrier departs $\hat{\mathbf{S}}_{1}$ with group velocity $\mathbf{v}_{\mathrm{N}}$ and $\operatorname{spin}\left|\mathbf{e}_{\mathrm{N}}\right\rangle$ and arrives at $\hat{\mathbf{S}}_{2}$ with a different group velocity $\mathbf{v}_{P}$ and spin $\left|\mathbf{e}_{\mathrm{P}}\right\rangle$, as described by $\hat{G}\left(\mathbf{R}_{2}, \mathbf{R}_{1}, E_{F}\right) \propto\left|\mathbf{e}_{\mathrm{P}}\right\rangle\left\langle\mathbf{e}_{\mathrm{N}}\right|$. Next, the contact exchange interaction with $\hat{\mathbf{S}}_{2}$ reverses the carrier group velocity to $-\mathbf{v}_{\mathrm{P}}$ and the carrier spin to $\left|-\mathbf{e}_{\mathrm{P}}\right\rangle$. It also increases $\hat{\mathbf{S}}_{2} \cdot \mathbf{e}_{\mathrm{P}}$ by one to conserve the total spin along $\mathbf{e}_{\mathrm{P}}$.

(2) This carrier departs $\hat{\mathbf{S}}_{2}$ with group velocity $-\mathbf{v}_{P}$ and spin $\left|-\mathbf{e}_{\mathrm{P}}\right\rangle$ and travels back to $\hat{\mathbf{S}}_{1}$ with a different group velocity $-\mathbf{v}_{\mathrm{N}}$ and spin $\left|-\mathbf{e}_{\mathrm{N}}\right\rangle$, as described by $\hat{G}\left(\mathbf{R}_{1}, \mathbf{R}_{2}, E_{F}\right) \propto \mid-$ $\left.\mathbf{e}_{\mathrm{N}}\right\rangle\left\langle-\mathbf{e}_{\mathrm{P}}\right|$. Next, the contact exchange interaction with $\hat{\mathbf{S}}_{1}$ restores its initial velocity $\mathbf{v}_{\mathrm{N}}$ and initial spin $\left|\mathbf{e}_{\mathrm{N}}\right\rangle$. It also decreases $\hat{\mathbf{S}}_{1} \cdot \mathbf{e}_{\mathrm{N}}$ by one to conserve the total spin along $\mathbf{e}_{\mathrm{N}}$.

We use a complex vector $\mathbf{g}=(0,1, i \sin \theta)$ and $g_{0} \equiv(\mathbf{g} \cdot \mathbf{g})^{1 / 2}$ to characterize the spin polarization: $\left|\mathbf{e}_{\mathrm{P}}\right\rangle\left\langle\mathbf{e}_{\mathrm{N}}\right|=\left(g_{0}+\hat{\boldsymbol{\sigma}} \cdot \mathbf{g}\right) / 2$, then Eq. (6) becomes

$$
\hat{H}_{\mathrm{RKKY}} \approx-\frac{J_{0} t^{2}}{R_{N}+R_{P}} \frac{2 \pi}{\Phi} \operatorname{Im} e^{2 i \Phi}\left(g_{0}^{2} \hat{\mathbf{S}}_{1} \cdot \hat{\mathbf{S}}_{2}+i g_{0} \mathbf{g} \cdot \hat{\mathbf{D}}-\mathbf{g} \cdot \mathbb{A} \cdot \mathbf{g}\right)
$$

which agrees with our numerical results in Fig. 2 at $\left|\mathbf{R}_{2}-\tilde{\mathbf{R}}_{1}\right| \gtrsim$ $\lambda_{F}$, e.g., the enhancement of the RKKY interaction near the focal point originates from $2 \pi / \Phi$, while the distinct angular dependencies of different interactions are well described by:

$$
\left\{\begin{array} { r l } 
{ \hat { \mathbf { S } } _ { 1 } \cdot \hat { \mathbf { S } } _ { 2 } } & { \sim \operatorname { c o s } ^ { 5 } \varphi , } \\
{ \hat { S } _ { 1 y } \hat { S } _ { 2 y } } & { \sim \operatorname { c o s } ^ { 3 } \varphi , } \\
{ \hat { D } _ { y } } & { \sim \operatorname { c o s } ^ { 4 } \varphi , }
\end{array} \quad \left\{\begin{array}{rl}
\hat{S}_{1 z} \hat{S}_{2 z} & \sim \cos ^{3} \varphi \sin ^{2} \varphi \\
\hat{D}_{z} & \sim \cos ^{4} \varphi \sin \varphi \\
\hat{A}_{y z} & \sim \cos ^{3} \varphi \sin \varphi
\end{array}\right.\right.
$$

where $\varphi$ is the polar angle of $\mathbf{R}_{2}-\tilde{\mathbf{R}}_{1}$.

For comparison, on a uniform N-type TI surface with Fermi momentum $q_{F}$, the classical trajectory going from $\hat{\mathbf{S}}_{1}$ to $\hat{\mathbf{S}}_{2}$ is a straight line with a length $R \equiv\left|\mathbf{R}_{2}-\mathbf{R}_{1}\right|$ [cf. Fig. [1(a)], so the propagator is obtained from Eq. (4) by setting $t=1$, $\mathbf{e}_{\mathrm{N}}=\mathbf{e}_{\mathrm{P}}=\mathbf{e} \propto \mathbf{e}_{z} \times\left(\mathbf{R}_{2}-\mathbf{R}_{1}\right)$, and $\Phi=q_{F} R$, while the RKKY interaction is obtained from Eq. (6) by further replacing $R_{N}+R_{P}$ with $R$. Compared with their counterparts on a uniform TI surface, Eqs. (4) and (6) exhibit two distinguishing features. First, the carrier propagators depend on $\mathbf{R}_{2}-\tilde{\mathbf{R}}_{1}$ instead of $\mathbf{R}_{2}-\mathbf{R}_{1}$, as if $\hat{\mathbf{S}}_{1}$ located at $\tilde{\mathbf{R}}_{1}$ instead of $\mathbf{R}_{1}$. This spatial symmetry is absent from the system Hamiltonian. Due to this symmetry, when $\left|\mathbf{R}_{2}-\tilde{\mathbf{R}}_{1}\right|$ is fixed, the RKKY interaction in Eq. (6) decays as $1 /\left(R_{N}+R_{P}\right)$, in contrast to the much faster $1 / R^{d}$ decay in uniform $d$-dimensional systems. Second, a carrier departs $\hat{\mathbf{S}}_{1}$ with spin orientation $\mathbf{e}_{\mathrm{N}}$ and arrives at $\hat{\mathbf{S}}_{2}$ with a different spin orientation $\mathbf{e}_{\mathrm{P}}$. This lifts the constraint of total spin conservation along $\mathbf{e}$ on a uniform TI surface [cf. Eq. (3)]. Interestingly, $\mathbf{e}_{\mathrm{N}}$ and $\mathbf{e}_{\mathrm{P}}$ are symmetric about the $x$ axis. This spin symmetry is also absent from the system Hamiltonian. Due to this symmetry, Eq. (7) only contains six terms as shown in Fig. 2, other terms are high-order contributions beyond the stationary phase approximation and hence are much smaller. This hidden spatial symmetry and spin symmetry arise from the mirror reflection symmetry of the electron and hole Fermi contours of a symmetric P-N junction ${ }^{77}$.

The results above are valid for $\hat{\mathbf{S}}_{2}$ far from $\tilde{\mathbf{R}}_{1}$, otherwise

$$
\hat{G}\left(\mathbf{R}_{2}, \mathbf{R}_{1}, E_{F}\right)=\frac{q_{F}}{4 \pi i v_{F}}\left(\tilde{g}_{0}+\hat{\boldsymbol{\sigma}} \cdot \tilde{\mathbf{g}}\right),
$$

where $\tilde{g}_{0}$ and $\tilde{\mathbf{g}} \equiv\left(0, \tilde{g}_{y}, \tilde{g}_{z}\right)$ are oscillatory functions of $q_{F}\left(\mathbf{R}_{2}-\tilde{\mathbf{R}}_{1}\right.$ ) (see Appendix A). Then Eq. (5) gives

$$
\hat{H}_{\mathrm{RKKY}} \approx-\frac{J_{0}}{2 a} \operatorname{Re}\left(\frac{\tilde{g}_{0}^{2}+\tilde{\mathbf{g}} \cdot \tilde{\mathbf{g}}}{2} \hat{\mathbf{S}}_{1} \cdot \hat{\mathbf{S}}_{2}+i \tilde{g} 0 \tilde{\mathbf{g}} \cdot \hat{\mathbf{D}}-\tilde{\mathbf{g}} \cdot \mathbb{A} \cdot \tilde{\mathbf{g}}\right) .
$$

Equations (7) and (9) are complementary and provide a full description for the spatial map of the RKKY interaction. When $\mathbf{R}_{2}-\tilde{\mathbf{R}}_{1}$ and hence $\tilde{g}_{0}, \tilde{\mathbf{g}}$ are fixed, the RKKY interaction follows $1 / R$ decay, instead of the much faster $1 / R^{d}$ decay in $d$-dimensional uniform systems. When $\hat{\mathbf{S}}_{2}$ locates at the focal point $\tilde{\mathbf{R}}_{1}$, we have $\tilde{g}_{0}=\pi / 2, \tilde{g}_{y}=2$, and $\tilde{g}_{z}=0$, so

$$
\hat{H}_{\mathrm{RKKY}} \approx-\frac{J_{0}}{2 a}\left(\frac{\pi^{2} / 4+4}{2} \hat{\mathbf{S}}_{1} \cdot \hat{\mathbf{S}}_{2}-4 \hat{S}_{1}^{y} \hat{S}_{2}^{y}\right) .
$$

Compared with a uniform TI surface with the same carrier concentration $^{35}$, the P-N interface amplifies the RKKY interaction by a dramatic factor $\sim 2 R / \lambda_{F}$ that increases with increasing distance $R=2 a$.

\section{B. Asymmetric P-N junction $\left(E_{F} \neq 0\right)$}

Here $q_{N} \neq q_{P}$, so the $\mathrm{P}-\mathrm{N}$ junction is characterized by two parameters: $q_{F} \equiv\left(q_{N}+q_{P}\right) / 2=V_{0} / v_{F}$ (or equivalently $\left.\lambda_{F} \equiv 2 \pi / q_{F}\right)$ controls the average carrier density, while the effective "refractive" index $n \equiv q_{P} / q_{N}$ controls the degree of 


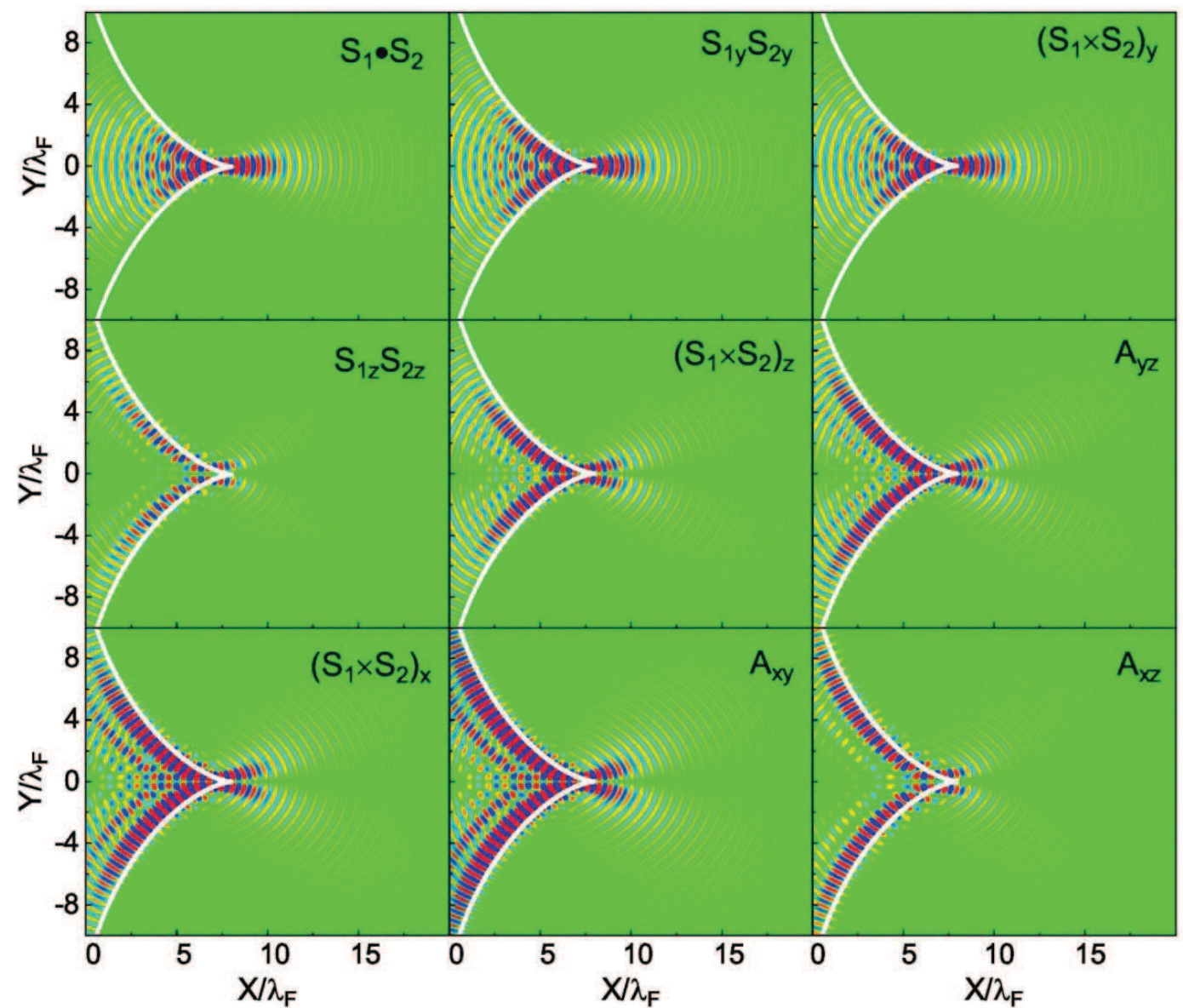

-0.3
0.0

0.3

$-0.1$

0.0

0.1

$-0.01$

0.00

0.01

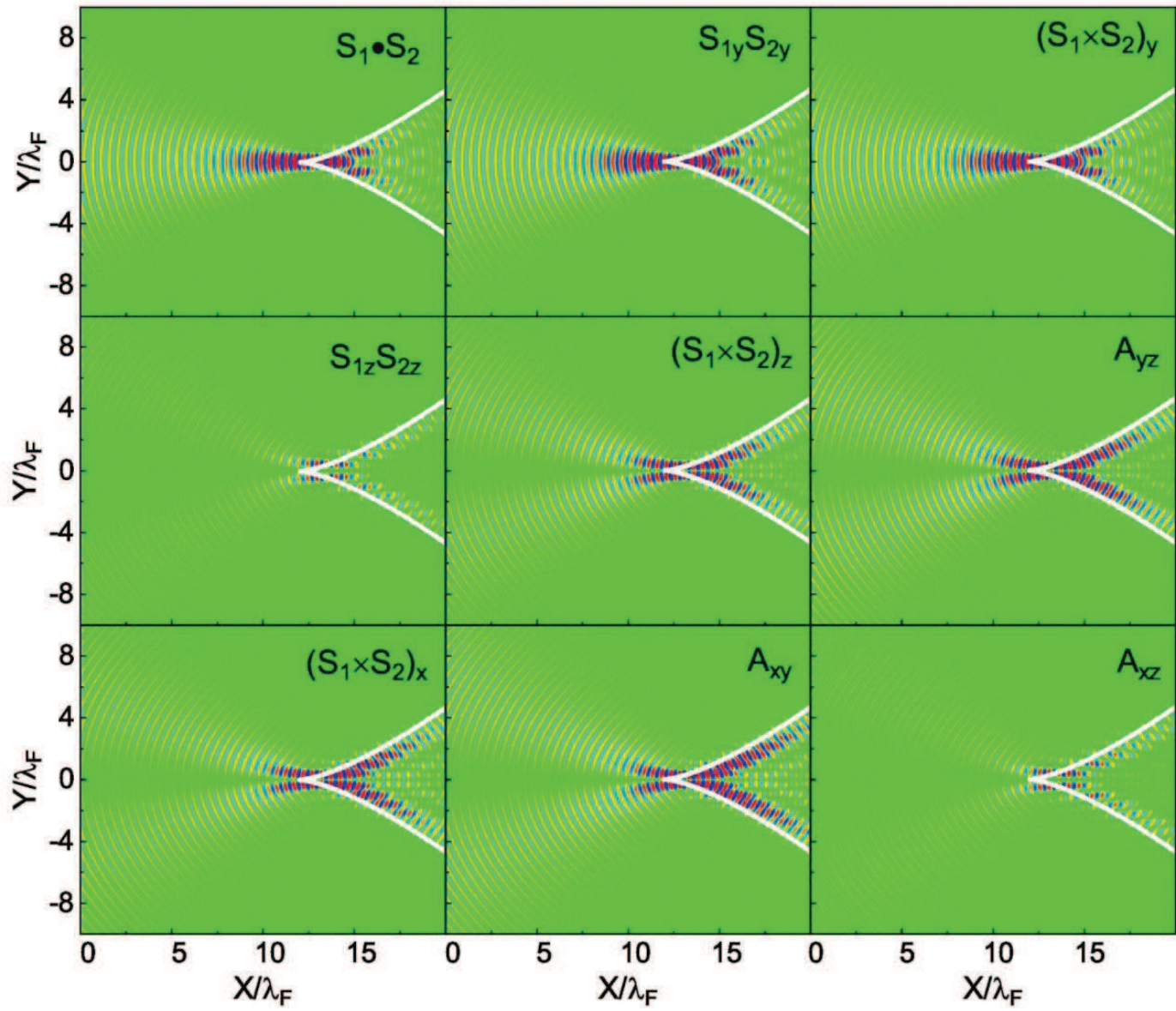

$-0.3$

0.0

0.3

$-0.1$

$-0.1$

0.0

0.1

$-0.01$

0.00

0.01

FIG. 3. Numerical results for the spatial map of the RKKY interactions [unit: $J_{0} /(2 a)$ ] as functions of $\mathbf{R}_{2}=(X, Y)$ in the P region of a P-N junction. The first spin is fixed at $\mathbf{R}_{1}=(-a, 0)=\left(-10 \lambda_{F}, 0\right)$. The white lines indicate the caustics. Upper part: $E_{F}=V_{0} / 9$ and hence $n=0.8$. Lower part: $E_{F}=-V_{0} / 11$ and hence $n=1.2$. 
asymmetry of the junction. As shown in Fig. 1(c), the incident and refractive trajectories are no longer mirror symmetric about the P-N interface. The electrons emanating from $\hat{\mathbf{S}}_{1}$ are refocused imperfectly by the $\mathrm{P}-\mathrm{N}$ interface, giving rise to caustics [black curve in Fig. 1(c)] ${ }^{57}$, as described by

$$
\begin{aligned}
& Y_{\text {cau }}(X) \equiv \pm \frac{\left[(a n)^{2 / 3}-X^{2 / 3}\right]^{3 / 2}}{\sqrt{1-n^{2}}}(\text { for } n<1), \\
& Y_{\text {cau }}(X) \equiv \pm \frac{\left[X^{2 / 3}-(n a)^{2 / 3}\right]^{3 / 2}}{\sqrt{n^{2}-1}}(\text { for } n>1) .
\end{aligned}
$$

For weak asymmetry (e.g., $n=0.8$ and $n=1.2$ ), the RKKY interactions are shown in Fig. 3. For $n=0.8(n=1.2)$, all the interactions are enhanced when $\hat{\mathbf{S}}_{2}$ locates in the left (right) neighborhood of the caustics, but are suppressed in the right (left) neighborhood. Moreover, the interactions $\hat{D}_{x}, \hat{A}_{x y}$, and $\hat{A}_{x z}$ are significantly smaller than other terms. Next, we present analytical expressions that reproduce these features.

As shown in Fig. 1. (c), the classical trajectory from $\hat{\mathbf{S}}_{1}$ to $\hat{\mathbf{S}}_{2}$ is characterized by an incident angle $\theta_{N}$, refractive angle $\theta_{P}$, transmission amplitude $t=\cos \theta_{N} / \cos \left[\left(\theta_{P}-\theta_{N}\right) / 2\right]$, and trajectory length $R_{N} \equiv a / \cos \theta_{N}\left(R_{P} \equiv X / \cos \theta_{P}\right)$ in the $\mathrm{N}(\mathrm{P})$ region, so that the total propagation phase is $\Phi=q_{N} R_{N}-q_{P} R_{P}$. Here $\theta_{N}$ and $\theta_{P}$ are determined by the momentum conservation along the $y$ axis, $\sin \theta_{N}=n \sin \theta_{P}$, and the geometric constraint $Y=a \tan \theta_{N}-X \tan \theta_{P}$. For $\hat{\mathbf{S}}_{2}$ far from the caustics, the propagator is dominated by a single classical trajectory (see Appendix B):

$$
\hat{G}\left(\mathbf{R}_{2}, \mathbf{R}_{1}, E_{F}\right) \approx t \frac{q_{F}}{i v_{F}} \frac{e^{i \Phi}}{\sqrt{2 \pi i q_{F} \tilde{R}}}\left|\mathbf{e}_{\mathrm{P}}\right\rangle\left\langle\mathbf{e}_{\mathrm{N}}\right|,
$$

where $\mathbf{e}_{\mathrm{N}}, \mathbf{e}_{\mathrm{P}}$ are shown in Fig. 11(c), and

$$
\tilde{R} \equiv \frac{1+n}{2} R_{N}-\frac{1+1 / n}{2} \frac{\cos ^{2} \theta_{N}}{\cos ^{2} \theta_{P}} R_{P}
$$

Taking the time reversal of the carrier spin (i.e., $\left|\mathbf{e}_{\mathrm{P}}\right\rangle\left\langle\mathbf{e}_{\mathrm{N}}\right| \rightarrow$ $\left.\left|-\mathbf{e}_{\mathrm{N}}\right\rangle\left\langle-\mathbf{e}_{\mathrm{P}}\right|\right)$ gives the propagator from $\hat{\mathbf{S}}_{2}$ back to $\hat{\mathbf{S}}_{1}$. Substituting the propagators into Eq. (5) gives

$$
\hat{H}_{\mathrm{RKKY}} \approx-\frac{t^{2} J_{0}}{R_{N}+R_{P}} \frac{2 \pi}{q_{F} \tilde{R}} \operatorname{Im} e^{2 i \Phi} \hat{S}_{1, \mathbf{e}_{\mathrm{N}}}^{-} \hat{S}_{2, \mathrm{e}_{\mathrm{P}}}^{+} .
$$

The physical process leading to this interaction is similar to that for a symmetric P-N junction, except that $\mathbf{e}_{\mathrm{N}}$ and $\mathbf{e}_{\mathrm{P}}$ are no longer symmetric about the $x$ axis.

We use a complex vector $\mathbf{g}=\left(\sin \left[\left(\theta_{P}-\theta_{N}\right) / 2\right], \cos \left[\left(\theta_{P}-\right.\right.\right.$ $\left.\left.\left.\left.\theta_{N}\right) / 2\right], i \sin \left[\theta_{N}+\theta_{P}\right) / 2\right]\right)$ and $g_{0}=(\mathbf{g} \cdot \mathbf{g})^{1 / 2}$ to characterize the spin polarization: $\left|\mathbf{e}_{\mathrm{P}}\right\rangle\left\langle\mathbf{e}_{\mathrm{N}}\right|=\left(g_{0}+\hat{\boldsymbol{\sigma}} \cdot \mathbf{g}\right) / 2$, then

$\hat{H}_{\mathrm{RKKY}} \approx-\frac{J_{0} t^{2}}{R_{N}+R_{P}} \frac{2 \pi}{q_{F} \tilde{R}} \operatorname{Im} e^{2 i \Phi}\left(g_{0}^{2} \hat{\mathbf{S}}_{1} \cdot \hat{\mathbf{S}}_{2}+i g_{0} \mathbf{g} \cdot \hat{\mathbf{D}}-\mathbf{g} \cdot \mathbb{A} \cdot \mathbf{g}\right)$,

which agrees with our numerical results in Fig. 3 as long as $\hat{\mathbf{S}}_{2}$ is away from the caustics by a distance $\gtrsim \lambda_{F}$, see Fig. 4 for a comparison. For weak asymmetry, i.e., $\left|E_{F}\right| \ll V_{0}$ and hence $n \approx 1$, the difference $\theta_{N}-\theta_{P}$ is small, so the polarization vector $\mathbf{g}$ has a small $x$ component. This explains why $D_{x}$ and $A_{x y}, A_{x z}$ in Fig. 3 are significantly smaller than other terms.
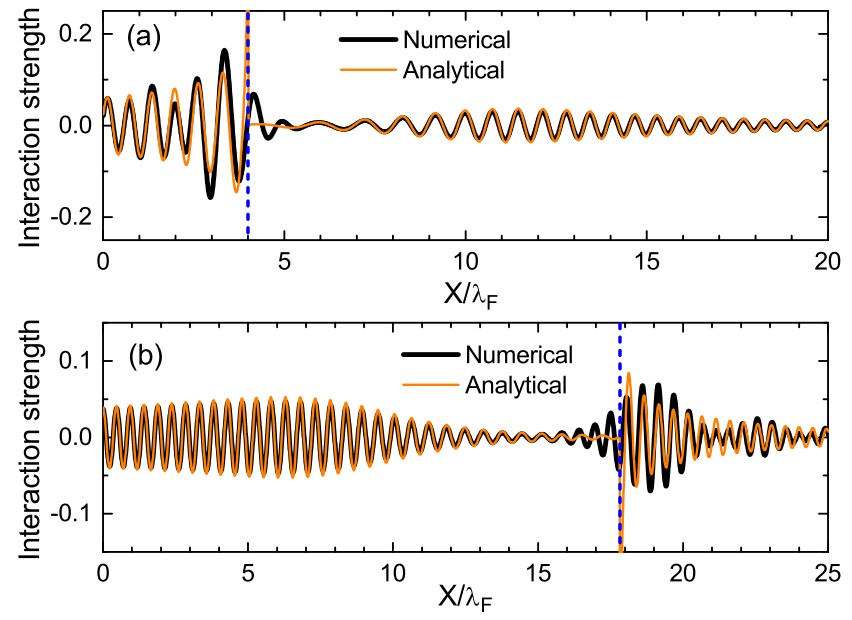

FIG. 4. Strength of the interaction $\hat{A}_{y z}$ [unit: $J_{0} /(2 a)$ ] as a function of $X$ (with $Y=3 \lambda_{F}$ fixed) in an asymmetric TI P-N junction with (a) $n=0.8$ and (b) $n=1.2$. The vertical gray lines marks the caustics.

\section{Selective generation and amplification of RKKY interactions}

The TI P-N junction is controlled by two parameters: (i) The junction voltage $V_{0}$ or equivalently the average Fermi momentum $q_{F} \equiv V_{0} / v_{F}$ (or $\lambda_{F} \equiv 2 \pi / q_{F}$ ) characterizes the average carrier density. (ii) The Fermi level $E_{F}$ or equivalently the "refractive" index $n$ characterizes the degree of asymmetry of the junction. In Eq. 12 , except for $J_{0} \propto q_{F}^{2}$ and $\Phi \propto q_{F}$, other quantities $\left(\theta_{N}, \theta_{P}, t, \tilde{R}, \mathbf{g}\right.$, and $\left.g_{0}\right)$ are completely determined by the locations of $\mathbf{S}_{1}$ and $\mathbf{S}_{2}$ and the "refraction" index $n$. Therefore, when the locations of $\mathbf{S}_{1}$ and $\mathbf{S}_{2}$ are fixed, $q_{F}$ controls the overall strength $(2 \pi / \tilde{\Phi}) J_{0} t^{2} /\left(R_{N}+R_{P}\right) \propto q_{F}$ of all the interactions, while $n$ controls the spin polarization vector $\mathbf{g}$ and hence the generation and selective amplification of different interactions.

Specifically, setting $n=1$ gives a symmetric $\mathrm{P}-\mathrm{N}$ junction with mirror symmetry on the Fermi contour, so $\theta_{N}=\theta_{P}=\theta$, $\mathbf{g}=(0,1, i \sin \theta)$, and the RKKY interaction is dominated by six terms [Eq. (7)]. Tuning $n$ slightly away from unity gives rise to small $\theta_{N}-\theta_{P}$ and hence $g_{x}$, which in turn generates three weak interactions $\hat{D}_{x}, \hat{A}_{x z}$, and $\hat{A}_{x y}$. Further tuning $n$ far from unity increases the degree of asymmetry of the P-N junction and hence further enhances the strengths of these interactions. As an example, we consider $a=5 \lambda_{F}, X=4 \lambda_{F}$, and $Y=2 \lambda_{F}$, and vary $E_{F} / V_{0}$ from -1 across zero to +1 to tune the "refraction" index $n$ from $+\infty$ across +1 to 0 . This variation changes the incident and refraction angles [Fig. 5(a)] and hence leads to two effects. First, it changes the effective distances $\tilde{R}$ and $R_{N}+R_{P}$ [Fig. 5(b)] and hence changes the overall strength of all the interactions, as shown in Fig. 5(c). Second, it changes the spin polarization vector $\mathbf{g}$ [Fig. 5(d)] and hence tunes the anisotropy of the RKKY interactions. According to Eq. (12), each interaction term oscillates rapidly as $\sin (2 \Phi)$ or $\cos (2 \Phi)$ with a corresponding envelope, so it is better to quantify the anisotropy of different interactions by these 

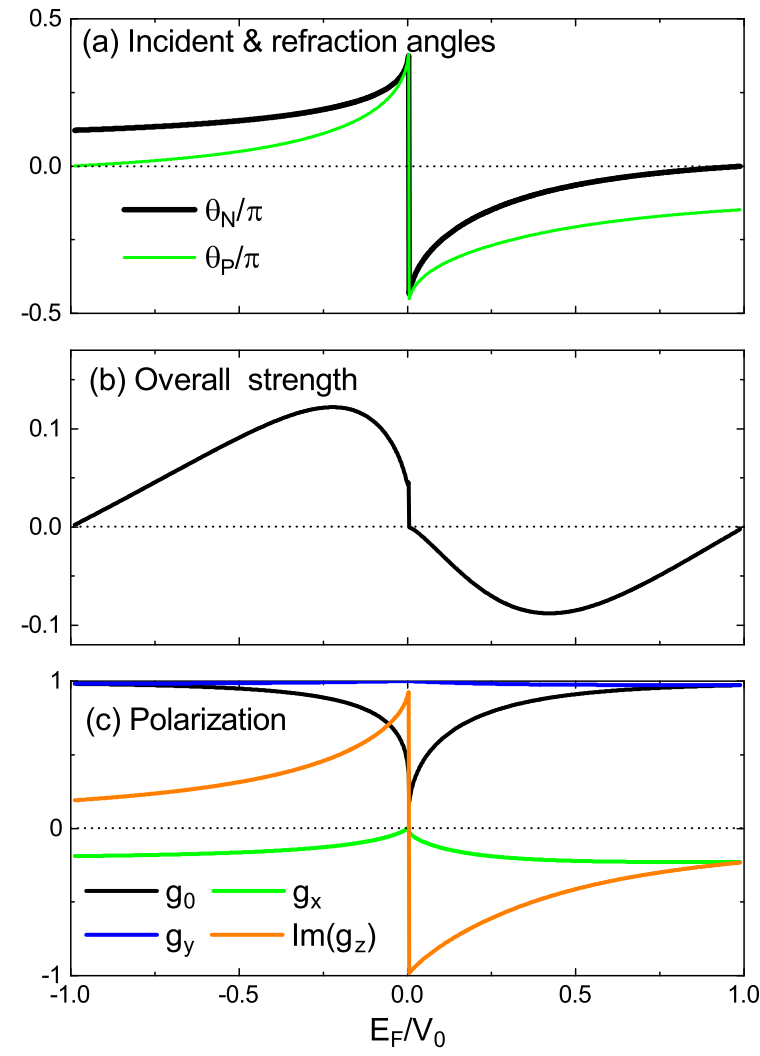
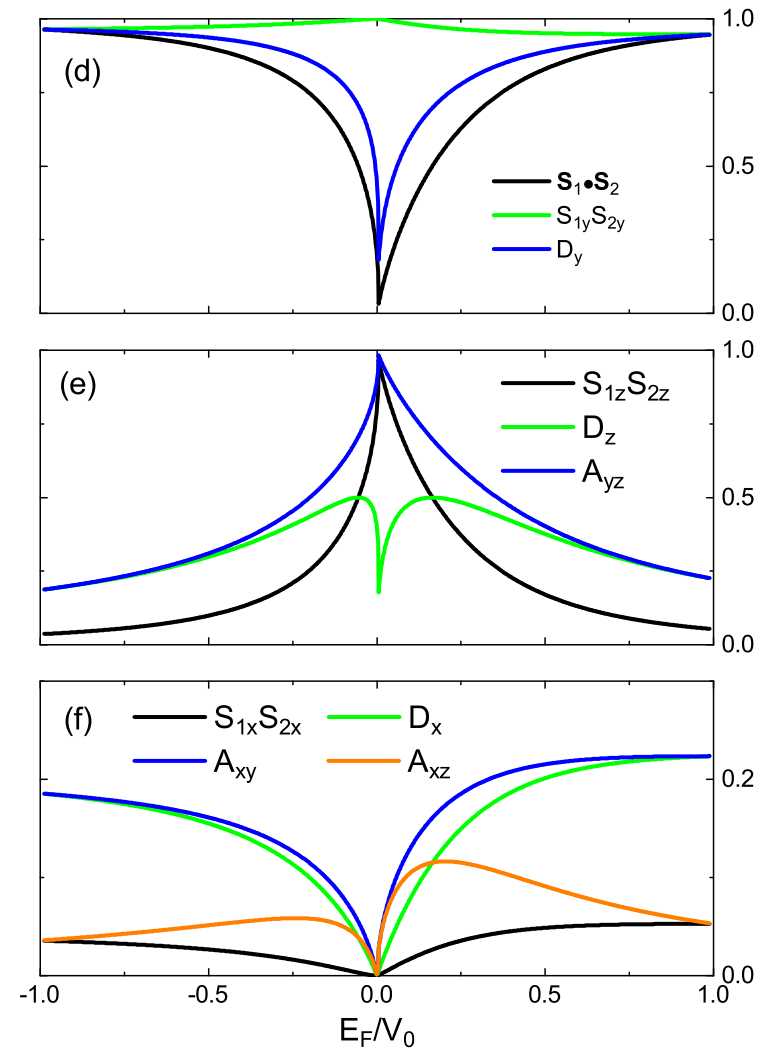

FIG. 5. (a) Incident and refraction angles, (b) overall strength $(2 \pi / \tilde{\Phi}) J_{0} t^{2} /\left(R_{N}+R_{P}\right)$ [unit: $J_{0} /(2 a)$ ], (c) spin polarization vector, and (d)(f) envelopes of different interaction terms [unit: $J_{0} /(2 a)$ ] as functions of $E_{F} / V_{0}$. The two local spins are fixed at $\mathbf{R}_{1}=\left(-5 \lambda_{F}, 0\right)$ and $\mathbf{R}_{2}=\left(4 \lambda_{F}, 2 \lambda_{F}\right)$. The dashed lines in (a)-(c) are guides to the eye.

envelopes. Then Fig. 5(d)-(f) clearly demonstrates the possibility to tune the relative strength of different interactions by tuning the "refraction" index.

\section{CONCLUSIONS}

We have proposed a physical mechanism for the selective generation and amplification of anisotropic RKKY interactions. The key is to utilize the negative refraction across a P-N junction to amplify all the interactions, and utilize the deflection of the carrier spin by the P-N interface (via the spin-orbit coupling) to achieve selected generation and amplification of anisotropic terms. Specifically, the junction potential $V_{0}$ controls the average carrier density and hence the overall strength of all the interactions, while the Fermi energy controls the degree of asymmetry of the P-N junction and hence the generation and amplification of anisotropic interactions. Although we have limited our numerical and analytical discussions to the surface of a TI P-N junction for the sake of specificity, this physical mechanism is applicable to the $\mathrm{P}-\mathrm{N}$ junction of an arbitrary system with spin-orbit coupling. Compared with previous works that focus on the dependence of the RKKY interaction on specific materials and carrier density, our work identifies the P-N interface as a second knob which, when combined with the carrier density, may open up the possibility for the independent control of the strength and anisotropy of the RKKY interaction in spin-orbit coupled systems.

\section{ACKNOWLEDGMENTS}

This work was supported by the National Key R\&D Program of China (Grants No. 2017YFA0303400), the MOST of China (Grants No. 2014CB848700), the NSFC (Grants No. 11774021, No. 11504018, and No. 11404043), and the NSFC program for "Scientific Research Center" (Grant No. U1530401). We acknowledge the computational support from the Beijing Computational Science Research Center (CSRC).

\section{Appendix A: Carrier propagators in symmetric TI P-N junction}

Given the carrier Hamiltonian $\hat{H}$, the propagator of the carriers is defined as $G_{\mu, v}\left(\mathbf{r}, \mathbf{r}_{0}, E_{F}\right) \equiv\left\langle\mathbf{r}, \mu\left|\left(E_{F}-\hat{H}+i 0^{+}\right)^{-1}\right| \mathbf{r}_{0}, v\right\rangle$, where $\mu, v=+$ (spin up) or - (spin down) denotes the spin states. When $\hat{H}$ is invariant under time-reversal operation $\hat{\theta}$, i.e., $\hat{\theta} \hat{H} \hat{\theta}^{-1}=\hat{H}$, we can use $\langle i|\hat{O}| j\rangle=\left\langle\hat{\theta} i\left|\hat{\theta} \hat{O} \hat{\theta}^{-1}\right| \hat{\theta} j\right\rangle^{*}=$ $\left\langle\hat{\theta} j\left|\left(\hat{\theta} \hat{O} \hat{\theta}^{-1}\right)^{\dagger}\right| \hat{\theta} i\right\rangle$ to obtain

$$
G_{\mu \nu}\left(\mathbf{r}, \mathbf{r}_{0}, E_{F}\right)=\left\langle\hat{\theta}\left(\mathbf{r}_{0}, \nu\right)\left|\left(E_{F}-\hat{H}+i 0^{+}\right)^{-1}\right| \hat{\theta}(\mathbf{r}, \mu)\right\rangle,
$$


where $|\hat{\theta}(\mathbf{r}, \pm)\rangle$ is the time-reversal of $|\mathbf{r}, \pm\rangle$. Using $\hat{\theta}|\mathbf{r}\rangle=\hat{\theta}|\mathbf{r}\rangle$ and $\hat{\theta}| \pm\rangle= \pm|\mp\rangle$, we obtain $|\hat{\theta}(\mathbf{r}, \pm)\rangle= \pm|\mathbf{r}, \mp\rangle$ and hence

$$
\begin{aligned}
& G_{\uparrow \uparrow}\left(\mathbf{r}, \mathbf{r}_{0}, E_{F}\right)=G_{\downarrow \downarrow}\left(\mathbf{r}_{0}, \mathbf{r}, E_{F}\right), \\
& G_{\downarrow \downarrow}\left(\mathbf{r}, \mathbf{r}_{0}, E_{F}\right)=G_{\uparrow \uparrow}\left(\mathbf{r}_{0}, \mathbf{r}, E_{F}\right), \\
& G_{\uparrow \downarrow}\left(\mathbf{r}, \mathbf{r}_{0}, E_{F}\right)=-G_{\downarrow \uparrow}\left(\mathbf{r}_{0}, \mathbf{r}, E_{F}\right), \\
& G_{\downarrow \uparrow}\left(\mathbf{r}, \mathbf{r}_{0}, E_{F}\right)=-G_{\uparrow \downarrow}\left(\mathbf{r}_{0}, \mathbf{r}, E_{F}\right) .
\end{aligned}
$$

Namely, given the $2 \times 2$ propagator $\hat{G}\left(\mathbf{r}, \mathbf{r}_{0}, E_{F}\right)=G_{0}+$ $\mathbf{G} \cdot \hat{\boldsymbol{\sigma}}$ from $\mathbf{r}_{0}$ to $\mathbf{r}$, the $2 \times 2$ propagator from $\mathbf{r}$ back to $\mathbf{r}_{0}$ can be obtained by taking time reversal of the carrier spin: $\hat{G}\left(\mathbf{r}_{0}, \mathbf{r}, E_{F}\right)=G_{0}-\mathbf{G} \cdot \hat{\boldsymbol{\sigma}}$. Therefore, in the following, we only consider the propagator from $\mathbf{R}_{1}$ to $\mathbf{R}_{2}$.

For convenience, we use $\mathbf{e}_{\varphi} \equiv \cos \varphi \mathbf{e}_{x}+\sin \varphi \mathbf{e}_{y}$ to denote a unit vector with polar angle $\varphi$. For $E_{F}=0$, the $\mathrm{N}$ region and the $\mathrm{P}$ region has the same Fermi momentum $q_{F} \equiv V_{0} / v_{F}$. We consider a right-going incident electron from the $\mathrm{N}$ region with an incident angle $\varphi$, momentum $\left(q_{x}, q_{y}\right) \equiv q_{F} \mathbf{e}_{\varphi}$, group velocity $\mathbf{v}_{\mathrm{N}} \equiv v_{F} \mathbf{e}_{\varphi}$, and spin state

$$
\left|\mathbf{e}_{z} \times \mathbf{v}_{\mathrm{N}}\right\rangle=\frac{1}{\sqrt{2}}\left[\begin{array}{l}
e^{-i \varphi / 2} \\
i e^{i \varphi / 2}
\end{array}\right],
$$

where $q_{x} \equiv\left(q_{F}^{2}-q_{y}^{2}\right)^{1 / 2}$. The reflection electron has a momentum $\left(-q_{x}, q_{y}\right) \equiv q_{F} \mathbf{e}_{\pi-\varphi}$, group velocity $\mathbf{v}_{\mathrm{r}}=v_{F} \mathbf{e}_{\pi-\varphi}$, and spin state

$$
\left|\mathbf{e}_{z} \times \mathbf{v}_{\mathbf{r}}\right\rangle=\frac{1}{\sqrt{2}}\left[\begin{array}{c}
e^{i \varphi / 2} \\
-i e^{-i \varphi / 2}
\end{array}\right]
$$

The transmission electron has a momentum $\left(-q_{x}, q_{y}\right)=$ $q_{F} \mathbf{e}_{\pi-\varphi}$, group velocity $\mathbf{v}_{\mathrm{P}} \equiv v_{F} \mathbf{e}_{-\varphi}$, and spin state

$$
\left|\mathbf{e}_{z} \times \mathbf{v}_{\mathrm{P}}\right\rangle=\frac{1}{\sqrt{2}}\left[\begin{array}{c}
e^{i \varphi / 2} \\
i e^{-i \varphi / 2}
\end{array}\right] .
$$

From the continuity equation at the P-N interface $x=0$,

$$
\left|\mathbf{e}_{z} \times \mathbf{v}_{\mathrm{N}}\right\rangle+r\left|\mathbf{e}_{z} \times \mathbf{v}_{\mathbf{r}}\right\rangle=t\left|\mathbf{e}_{z} \times \mathbf{v}_{\mathrm{P}}\right\rangle,
$$

we obtain the transmission amplitude $t(\varphi)=\cos \varphi$ and the reflection amplitude $r(\varphi)=i \sin \varphi$.

The carrier propagator from $\mathbf{R}_{1}=(-a, 0)$ in the $\mathrm{N}$ region to $\mathbf{R}_{2}=(X, Y)$ in the $\mathrm{P}$ region is given by

$$
\hat{G}\left(\mathbf{R}_{2}, \mathbf{R}_{1}, E_{F}\right)=\int_{-\infty}^{\infty} \frac{d q_{y}}{2 \pi} t(\varphi) e^{i \phi\left(q_{y}\right)} \frac{\left|\mathbf{e}_{z} \times \mathbf{v}_{\mathrm{P}}\right\rangle\left\langle\mathbf{e}_{z} \times \mathbf{v}_{\mathrm{N}}\right|}{i v_{F} q_{x} / q_{F}}
$$

where $\phi\left(q_{y}\right)=q_{y} Y+q_{x}(a-X)$. By keeping traveling waves only, we can replace $\int_{-\infty}^{\infty} d q_{y}$ by $\int_{-q_{F}}^{q_{F}} d q_{y}$ and obtain Eq. 8 in the main text, where

$$
\begin{aligned}
& \tilde{g}_{0} \equiv \int_{-\pi / 2}^{\pi / 2} \cos ^{2} \varphi e^{i \phi(\varphi)} d \varphi \\
& \tilde{g}_{y} \equiv \int_{-\pi / 2}^{\pi / 2} \cos \varphi e^{i \phi(\varphi)} d \varphi \\
& \tilde{g}_{z} \equiv i \int_{-\pi / 2}^{\pi / 2} \sin \varphi \cos \varphi e^{i \phi(\varphi)} d \varphi
\end{aligned}
$$
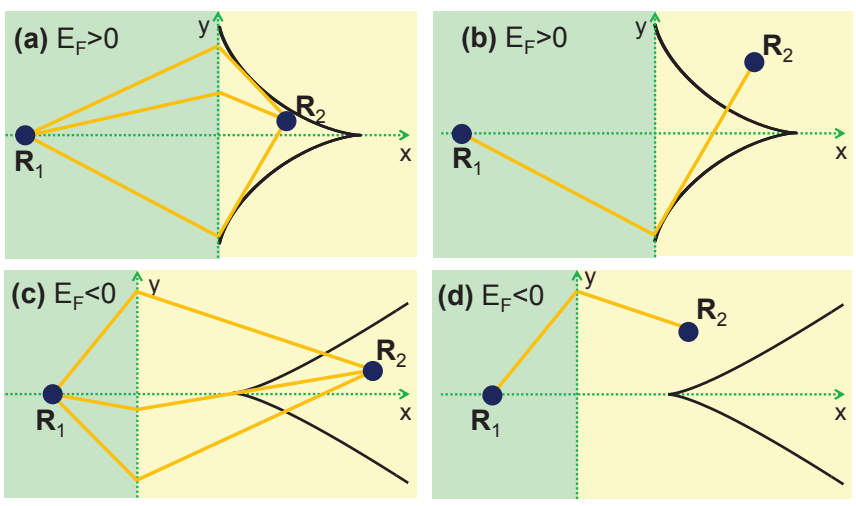

FIG. 6. Classical trajectory (orange lines) connecting $\mathbf{R}_{1}$ and $\mathbf{R}_{2}$ on opposite sides of a TI P-N junction. (a) and (b): $E_{F}>0$. (c) and (d): $E_{F}<0$. The black curves denote the caustics.

and $\phi(\varphi)=q_{F}[Y \sin \varphi+(a-X) \cos \varphi]$.

The incident angle $\theta$ of the classical trajectory is determined by $\partial_{\varphi} \phi(\varphi)=0$ as $\theta=\tan ^{-1}[Y /(a-X)]$. In terms of $\theta$ and the total propagation phase $\Phi \equiv q_{F}(a-X) / \cos \theta$ along the classical trajectory, we have $\phi(\varphi)=\Phi \cos (\varphi-\theta)$. For $\mathbf{R}_{2}$ far from the focal point $\tilde{\mathbf{R}}_{1} \equiv(a, 0)$, we can replace $\phi(\varphi)$ by its Taylor expansion near $\varphi=\theta$ up to the second order to obtain

$$
\left(\tilde{g}_{0}, \tilde{g}_{y}, \tilde{g}_{z}\right)=\cos \theta \sqrt{\frac{2 \pi}{i \Phi}} e^{i \Phi}(\cos \theta, 1, i \sin \theta)
$$

and hence Eq. (4) of the main text. For $\mathbf{R}_{2}=\tilde{\mathbf{R}}_{1} \equiv(a, 0)$ and hence $\phi(\varphi)=0$, we obtain $\tilde{g}_{0}=\pi / 2, \tilde{g}_{y}=2, \tilde{g}_{z}=0$. For $\mathbf{R}_{2}$ close to $\tilde{\mathbf{R}}_{1}$, we use the Jacobi-Anger expansion $e^{i z \cos \varphi}=$ $\sum_{n=-\infty}^{\infty} i^{n} J_{n}(z) e^{i n \varphi}$ to obtain

$$
\begin{aligned}
& \tilde{g}_{0} \equiv \frac{\pi}{2} J_{0}(\Phi)+\sum_{n \geq 1}^{\infty} i^{n} J_{n}(\Phi) \cos (n \theta)\left(\xi_{n-1}+\xi_{n+1}\right) \\
& \tilde{g}_{y} \equiv 2 J_{0}(\Phi)+2 \sum_{n \geq 1}^{\infty} i^{n} J_{n}(\Phi) \cos (n \theta) \xi_{|n|} \\
& \tilde{g}_{z} \equiv i \sum_{n \geq 1}^{\infty} i^{n} J_{n}(\Phi) \sin (n \theta)\left(\xi_{n-1}-\xi_{n+1}\right)
\end{aligned}
$$

where $J_{m}(x)$ is the $m$ th-order Bessel function and

$$
\xi_{ \pm n} \equiv \int_{-\pi / 2}^{\pi / 2} \cos \varphi e^{i n \varphi} d \varphi=\frac{-2 \cos (n \pi / 2)}{n^{2}-1}
$$

The value $\xi_{ \pm 1}=\pi / 2$ is obtained by taking the limit $n \rightarrow 1$.

\section{Appendix B: Carrier propagators in asymmetric TI P-N junction}

We consider a right-going incident electron with energy $E_{F}$ in the $\mathrm{N}$ region with an incident angle $\varphi_{N}$, momentum 
$\left(q_{N, x}, q_{y}\right) \equiv q_{N} \mathbf{e}_{\varphi_{N}}$, group velocity $\mathbf{v}_{\mathrm{N}} \equiv v_{F} \mathbf{e}_{\varphi_{N}}$, and spin state

$$
\left|\mathbf{e}_{z} \times \mathbf{v}_{\mathrm{N}}\right\rangle=\frac{1}{\sqrt{2}}\left[\begin{array}{l}
e^{-i \varphi_{N} / 2} \\
i e^{i \varphi_{N} / 2}
\end{array}\right]
$$

where $q_{N} \equiv\left(V_{0}+E_{F}\right) / v_{F}$ is the Fermi momentum in the $\mathrm{N}$ region and $q_{N, x} \equiv\left(q_{N}^{2}-q_{y}^{2}\right)^{1 / 2}$. The reflection electron has a momentum $\left(-q_{N, x}, q_{y}\right)=q_{N} \mathbf{e}_{\pi-\varphi_{N}}$, group velocity $\mathbf{v}_{\mathrm{r}}=v_{F} \mathbf{e}_{\pi-\varphi_{N}}$, and spin state

$$
\left|\mathbf{e}_{z} \times \mathbf{v}_{\mathbf{r}}\right\rangle=\frac{1}{\sqrt{2}}\left[\begin{array}{c}
e^{i \varphi_{N} / 2} \\
-i e^{-i \varphi_{N} / 2}
\end{array}\right]
$$

The transmission electron has a momentum $\left(-q_{P, x}, q_{y}\right)=$ $q_{P} \mathbf{e}_{\pi-\varphi_{P}}$, group velocity $\mathbf{v}_{\mathrm{P}} \equiv v_{F} \mathbf{e}_{-\varphi_{P}}$, and spin state

$$
\left|\mathbf{e}_{z} \times \mathbf{v}_{\mathrm{P}}\right\rangle=\frac{1}{\sqrt{2}}\left[\begin{array}{c}
e^{i \varphi_{P} / 2} \\
i e^{-i \varphi_{P} / 2}
\end{array}\right]
$$

where $q_{P} \equiv\left(V_{0}-E_{F}\right) / v_{F}$ is the Fermi momentum in the $\mathrm{P}$ region and $q_{P, x} \equiv\left(q_{P}^{2}-q_{y}^{2}\right)^{1 / 2}$. From the continuity equation at the P-N interface $x=0$,

$$
\left|\mathbf{e}_{z} \times \mathbf{v}_{\mathbf{N}}\right\rangle+r\left|\mathbf{e}_{z} \times \mathbf{v}_{\mathbf{r}}\right\rangle=t\left|\mathbf{e}_{z} \times \mathbf{v}_{\mathbf{P}}\right\rangle,
$$

we obtain the transmission amplitude $t\left(q_{y}\right)=$ $\cos \varphi_{N} / \cos \left[\left(\varphi_{P}-\varphi_{N}\right) / 2\right]$. The continuity of $q_{y}$ across the P-N interface dictates

$$
\frac{\sin \varphi_{N}}{\sin \varphi_{P}}=\frac{q_{P}}{q_{N}}=\frac{V_{0}-E_{F}}{V_{0}+E_{F}} \equiv n .
$$

The carrier propagator from $\mathbf{R}_{1}$ to $\mathbf{R}_{2}$ is

$$
\hat{G}\left(\mathbf{R}_{2}, \mathbf{R}_{1}, E_{F}\right)=\int \frac{d q_{y}}{2 \pi} t\left(q_{y}\right) e^{i \phi\left(q_{y}\right)} \frac{\left|\mathbf{e}_{z} \times \mathbf{v}_{\mathrm{P}}\right\rangle\left\langle\mathbf{e}_{z} \times \mathbf{v}_{\mathrm{N}}\right|}{i v_{F} q_{N, x} / q_{N}},
$$

where $\phi\left(q_{y}\right)=q_{y} Y+a q_{N, x}-q_{P, x} X$. The classical trajectory going from $\mathbf{R}_{1}$ to $\mathbf{R}_{2}$ is determined by $\partial_{q_{y}} \phi=0$ as

$$
Y=a \tan \varphi_{N}-X \tan \varphi_{P}
$$

which, together with Eq. (B1), determines the incident angle $\varphi_{N}$ and the refractive angle $\varphi_{P}$ for the classical trajectory. For clarity, we denote this classical incident (refractive) angle by $\theta_{N}\left(\theta_{P}\right)$. For $n<1(n>1)$, there is a unique solution when $\mathbf{R}_{2}$ lies on the right (left) of the caustics, or three solutions when $\mathbf{R}_{2}$ lies on the left (right) of the caustics, as shown schematically in Fig. 6. The contribution from different classical trajectories to the propagator are additive. Usually, as long as $\mathbf{R}_{2}$ is far from the caustics, the propagator is dominated by the middle trajectory. The contribution from this trajectory as characterized by $\left(\theta_{N}, \theta_{P}\right)$ is

$G\left(\mathbf{R}_{2}, \mathbf{R}_{1}, E_{F}\right) \approx \frac{t}{2 \pi i v_{F} \cos \theta_{N}}\left|\mathbf{e}_{z} \times \mathbf{v}_{\mathrm{P}}\right\rangle\left\langle\mathbf{e}_{z} \times \mathbf{v}_{\mathrm{N}}\right| \int_{-\infty}^{\infty} e^{i \phi\left(q_{y}\right)} d q_{y}$,

where $t=\cos \theta_{N} / \cos \left[\left(\theta_{P}-\theta_{N}\right) / 2\right]$ is the transmission amplitude of the classical trajectory. The propagation phase along the classical trajectory (i.e., $q_{y, \mathrm{c}}=q_{N} \sin \theta_{N}=q_{P} \sin \theta_{P}$ ) is

$$
\Phi \equiv \phi\left(q_{y, \mathrm{c}}\right)=q_{N} \frac{a}{\cos \theta_{N}}-q_{P} \frac{X}{\cos \theta_{P}} .
$$

Under the stationary phase approximation, we replace $\phi\left(q_{y}\right)$ by its second-order Taylor expansion

$$
\phi\left(q_{y}\right) \approx \Phi-\frac{1}{2} \frac{\tilde{R}}{q_{F} \cos ^{2} \theta_{N}}\left(q_{y}-q_{y, \mathrm{c}}\right)^{2}
$$

[ $\tilde{R}$ is given by Eq. 11 of the main text] to obtain Eq. 10 of the main text.
* wenyang@csrc.ac.cn

$\dagger$ kchang@semi.ac.cn

1 M. A. Ruderman and C. Kittel, Phys. Rev. 96, 99 (1954).

2 T. Kasuya, Prog. Theor. Phys. 16, 45 (1956).

3 K. Yosida, Phys. Rev. 106, 893 (1957).

4 T. Dietl and H. Ohno, Rev. Mod. Phys. 86, 187 (2014).

5 C. Piermarocchi, P. Chen, L. J. Sham, and D. G. Steel, Phys. Rev. Lett. 89, 167402 (2002).

${ }^{6}$ N. J. Craig, J. M. Taylor, E. A. Lester, C. M. Marcus, M. P. Hanson, and A. C. Gossard, Science 304, 565 (2004).

7 Y. Rikitake and H. Imamura, Phys. Rev. B 72, 033308 (2005).

${ }^{8}$ M. Friesen, A. Biswas, X. Hu, and D. Lidar, Phys. Rev. Lett. 98, 230503 (2007).

9 V. Srinivasa, H. Xu, and J. M. Taylor, Phys. Rev. Lett. 114, 226803 (2015).

10 D. Loss and D. P. DiVincenzo, Phys. Rev. A 57, 120 (1998).

${ }^{11}$ L. Trifunovic, O. Dial, M. Trif, J. R. Wootton, R. Abebe, A. Yacoby, and D. Loss, Phys. Rev. X 2, 011006 (2012).

12 S. A. Wolf, D. D. Awschalom, R. A. Buhrman, J. M. Daughton, S. von Molnar, M. L. Roukes, A. Y. Chtchelkanova, and D. M. Treger, Science 294, 1488 (2001).
13 I. Zutic, J. Fabian, and S. Das Sarma, Rev. Mod. Phys. 76, 323 (2004).

14 D. Pesin and A. H. MacDonald, Nat. Mater. 11, 409 (2012).

15 X. Liu, H.-C. Hsu, and C.-X. Liu, Phys. Rev. Lett. 111, 086802 (2013).

16 Q.-Z. Wang, X. Liu, H.-J. Zhang, N. Samarth, S.-C. Zhang, and C.-X. Liu, Phys. Rev. Lett. 113, 147201 (2014).

17 J. Klinovaja, P. Stano, A. Yazdani, and D. Loss, Phys. Rev. Lett. 111, 186805 (2013).

18 C. Jayaprakash, H. R. Krishna-murthy, and J. W. Wilkins, Phys. Rev. Lett. 47, 737 (1981).

19 B. A. Jones and C. M. Varma, Phys. Rev. Lett. 58, 843 (1987).

${ }^{20}$ B. A. Jones, C. M. Varma, and J. W. Wilkins, Phys. Rev. Lett. 61, 125 (1988).

${ }^{21}$ P. Gegenwart, Q. Si, and F. Steglich, Nat. Phys. 4, 186 (2008).

${ }^{22}$ N. Néel, R. Berndt, J. Kröger, T. O. Wehling, A. I. Lichtenstein, and M. I. Katsnelson, Phys. Rev. Lett. 107, 106804 (2011).

${ }^{23}$ H. Pruser, P. E. Dargel, M. Bouhassoune, R. G. Ulbrich, T. Pruschke, S. Lounis, and M. Wenderoth, Nat. Commun. 5, 5417 (2014).

24 A. Spinelli, M. Gerrits, R. Toskovic, B. Bryant, M. Ternes, and 
A. F. Otte, Nat. Commun. 6, 10046 (2015).

25 M. A. H. Vozmediano, M. P. López-Sancho, T. Stauber, and F. Guinea, Phys. Rev. B 72, 155121 (2005).

26 V. K. Dugaev, V. I. Litvinov, and J. Barnas, Phys. Rev. B 74, 224438 (2006).

${ }^{27}$ L. Brey, H. A. Fertig, and S. Das Sarma, Phys. Rev. Lett. 99, 116802 (2007)

28 S. Saremi, Phys. Rev. B 76, 184430 (2007).

${ }^{29}$ E. H. Hwang and S. Das Sarma, Phys. Rev. Lett. 101, 156802 (2008).

30 B. Uchoa, T. G. Rappoport, and A. H. Castro Neto, Phys. Rev. Lett. 106, 016801 (2011).

${ }^{31}$ H.-R. Chang, J. Zhou, S.-X. Wang, W.-Y. Shan, and D. Xiao, Phys. Rev. B 92, 241103(R) (2015).

32 M. V. Hosseini and M. Askari, Phys. Rev. B 92, 224435 (2015).

33 Q. Liu, C.-X. Liu, C. Xu, X.-L. Qi, and S.-C. Zhang, Phys. Rev. Lett. 102, 156603 (2009).

34 R. R. Biswas and A. V. Balatsky, Phys. Rev. B 81, 233405 (2010).

35 J.-J. Zhu, D.-X. Yao, S.-C. Zhang, and K. Chang, Phys. Rev. Lett. 106, 097201 (2011).

36 D. A. Abanin and D. A. Pesin, Phys. Rev. Lett. 106, 136802 (2011).

37 D. K. Efimkin and V. Galitski, Phys. Rev. B 89, 115431 (2014).

38 V. I. Litvinov, Phys. Rev. B 89, 235316 (2014).

39 A. A. Zyuzin and D. Loss, Phys. Rev. B 90, 125443 (2014).

40 P. Wahl, P. Simon, L. Diekhöner, V. S. Stepanyuk, P. Bruno, M. A. Schneider, and K. Kern, Phys. Rev. Lett. 98, 056601 (2007).

${ }^{41}$ F. Meier, L. Zhou, J. Wiebe, and R. Wiesendanger, Science 320, 82 (2008).

${ }^{42}$ L. Zhou, J. Wiebe, S. Lounis, E. Vedmedenko, F. Meier, S. Blugel, P. H. Dederichs, and R. Wiesendanger, Nat. Phys. 6, 187 (2010).

43 A. A. Khajetoorians, J. Wiebe, B. Chilian, S. Lounis, S. Blugel, and R. Wiesendanger, Nat. Phys. 8, 497 (2012).

44 O. O. Brovko, P. A. Ignatiev, V. S. Stepanyuk, and P. Bruno, Phys. Rev. Lett. 101, 036809 (2008).

45 D. S. Inosov, D. V. Evtushinsky, A. Koitzsch, V. B. Zabolotnyy, S. V. Borisenko, A. A. Kordyuk, M. Frontzek, M. Loewenhaupt, W. Löser, I. Mazilu, et al., Phys. Rev. Lett. 102, 046401 (2009).

${ }^{46}$ F. Xiu, Y. Wang, J. Kim, A. Hong, J. Tang, A. P. Jacob, J. Zou, and K. L. Wang, Nat. Mater. 9, 337 (2010).

47 A. M. Black-Schaffer, Phys. Rev. B 82, 073409 (2010).

48 S. R. Power, P. D. Gorman, J. M. Duffy, and M. S. Ferreira, Phys. Rev. B 86, 195423 (2012).

49 S. R. Power, F. S. M. Guimarães, A. T. Costa, R. B. Muniz, and M. S. Ferreira, Phys. Rev. B 85, 195411 (2012).

${ }^{50}$ H. Chen, Q. Niu, Z. Zhang, and A. H. MacDonald, Phys. Rev. B 87, 144410 (2013)

${ }^{51}$ N. Y. Yao, L. I. Glazman, E. A. Demler, M. D. Lukin, and J. D. Sau, Phys. Rev. Lett. 113, 087202 (2014).

52 M. Bouhassoune, B. Zimmermann, P. Mavropoulos, D. Wort- mann, P. H. Dederichs, S. Blgel, and S. Lounis, Nat. Commun. 5, 5558 (2014)

53 N. Klier, S. Shallcross, S. Sharma, and O. Pankratov, Phys. Rev. B 92, 205414 (2015).

54 T. Nie, J. Tang, X. Kou, Y. Gen, S. Lee, X. Zhu, Q. He, L.-T. Chang, K. Murata, Y. Fan, et al., Nat. Commun. 7, 12866 (2016).

55 S.-H. Zhang, J.-J. Zhu, W. Yang, and K. Chang, 2D Materials 4 , 035005 (2017).

56 J.-J. Zhu, K. Chang, R.-B. Liu, and H.-Q. Lin, Phys. Rev. B 81, 113302 (2010).

57 V. V. Cheianov, V. Fal'ko, and B. L. Altshuler, Science 315, 1252 (2007).

${ }^{58}$ L. Zhao, P. Tang, B.-L. Gu, and W. Duan, Phys. Rev. Lett. 111, 116601 (2013).

59 G.-H. Lee, G.-H. Park, and H.-J. Lee, Nat. Phys. 11, 925 (2015).

${ }^{60}$ S. Chen, Z. Han, M. M. Elahi, K. M. M. Habib, L. Wang, B. Wen, Y. Gao, T. Taniguchi, K. Watanabe, J. Hone, et al., Science 353, 1522 (2016).

61 J. Wang, X. Chen, B.-F. Zhu, and S.-C. Zhang, Phys. Rev. B 85, 235131 (2012).

${ }^{62}$ K. M. M. Habib, R. N. Sajjad, and A. W. Ghosh, Phys. Rev. Lett. 114, 176801 (2015).

${ }^{63}$ R. Ilan, F. de Juan, and J. E. Moore, Phys. Rev. Lett. 115, 096802 (2015).

${ }^{64}$ Y. Li, J. Zhang, G. Zheng, Y. Sun, S. S. Hong, F. Xiong, S. Wang, H. R. Lee, and Y. Cui, ACS Nano 9, 10916 (2015).

65 T. Bathon, S. Achilli, P. Sessi, V. A. Golyashov, K. A. Kokh, O. E. Tereshchenko, and M. Bode, Advanced Materials 28, 2183 (2016).

66 N. H. Tu, Y. Tanabe, Y. Satake, K. K. Huynh, and K. Tanigaki, Nat. Commun. 7, 13763 (2016).

${ }^{67}$ S. H. Kim, K.-H. Jin, B. W. Kho, B.-G. Park, F. Liu, J. S. Kim, and H. W. Yeom, ACS Nano 11, 9671 (2017).

${ }^{68}$ P. Bruno, Phys. Rev. B 52, 411 (1995).

69 L. M. Roth, H. J. Zeiger, and T. A. Kaplan, Phys. Rev. 149, 519 (1966).

${ }^{70}$ Q. Liu, X.-L. Qi, and S.-C. Zhang, Phys. Rev. B 85, 125314 (2012).

71 S. R. Power and M. S. Ferreira, Phys. Rev. B 83, 155432 (2011).

72 M. Z. Hasan and C. L. Kane, Rev. Mod. Phys. 82, 3045 (2010).

73 X.-L. Qi and S.-C. Zhang, Rev. Mod. Phys. 83, 1057 (2011).

${ }^{74}$ Q. Tong, H. Yu, Q. Zhu, Y. Wang, X. Xu, and W. Yao, Nature Physics 13, 356 (2016).

75 V. G. Veselago, Soviet Physics Uspekhi 10, 509 (1968).

76 S. Lounis, P. Zahn, A. Weismann, M. Wenderoth, R. G. Ulbrich, I. Mertig, P. H. Dederichs, and S. Blügel, Phys. Rev. B 83, 035427 (2011).

77 S.-H. Zhang, J.-J. Zhu, W. Yang, H.-Q. Lin, and K. Chang, Phys. Rev. B 94, 085408 (2016). 